Turkish Journal of Geriatrics

DOI: $10.31086 /$ tigeri.2020.136

2020; 23(1): $42-50$

n Aylin GÜNEŞLi' ${ }^{1}$ (D)

- Mustafa YILMAZ2 (D)

- Çiğdem YALÇIN ${ }^{1}$ (D)

- Nihan TEKKARIŞMAZ ${ }^{3}$ D

- Özlem ALKAN ${ }^{1}$ (D)

CORRESPONDANCE

Aylin GÜNEŞLI

Baskent University, Faculty of Medicine,

Department of Radiology, Adana, TURKEY.

Phone: +905333880221

e-mail: aylingunesli@hotmail.com

Received: 19/09/2019

Accepted:25/01/2020

\section{EVALUATION OF EPICARDIAL FAT AND CAROTID AND FEMORAL INTIMA-MEDIA THICKNESSES IN GERIATRIC PATIENTS WITH END-STAGE RENAL DISEASE}

\section{Abstract}

Introduction: Although atherosclerotic cardiovascular diseases and cardiovascular risks are known to increase in patients with end-stage renal disease, it is not clear whether these risks increase in the geriatric patient population as well. This study aims to evaluate these risks in geriatric patients with end-stage renal disease by evaluating epicardial fat and carotid and femoral intima-media thicknesses, known as markers, for subclinical atherosclerosis and cardiovascular risks.

Materials and Methods: This cross-sectional study included 52 patients who started to receive chronic hemodialysis treatment after the age of 65 years (mean age $73.92 \pm 5.63$ ) years with end-stage renal failure and 51 healthy volunteers (mean age: $74.49 \pm 4.63$ years). Epicardial fat and carotid and femoral intima-media thicknesses were measured and compared between these groups.

Results: Carotid intima-media and epicardial fat thicknesses were significantly higher in the patient group than in the control group $(0.91 \pm 0.08$ vs. $0.71 \pm 0.1$ $\mathrm{mm}, \mathrm{p}<0.001$ and $0.84 \pm 0.17$ vs. $0.75 \pm 0.17 \mathrm{~cm}, \mathrm{p}=0.01$, respectively). However, no significant difference was observed in femoral intima-media thickness between the two groups $(0.58 \pm 0.07$ vs. $0.56 \pm 0.97 \mathrm{~mm}, \mathrm{p}=0.266)$. Correlation analysis revealed a significant positive correlation between the duration of dialysis and epicardial fat and carotid intima-media thicknesses $(r=0.611, p<0.001$ and $r=0.337, p=0.015$, respectively). Furthermore, regression analysis revealed a significant relationship between the duration of dialysis and carotid intima-media thickness $(\beta=0.657$, $\mathrm{p}=0.001$ ).

Conclusion: Epicardial fat and carotid intima-media thicknesses increase in geriatric patients with end-stage renal disease but with no significant changes in femoral intima-media thickness, indirectly suggesting that subclinical atherosclerosis and cardiovascular risks are increased in these patients.

Keywords: Atherosclerosis; Echocardiography; Kidney disease; Ultrasonography.
Baskent University, Faculty of Medicine, Department of Radiology, Adana, TURKEY. Baskent University, Faculty of Medicine, Department of Cardiology, Adana, TURKEY. Baskent University, Faculty of Medicine, Department of Nephrology, Adana, TURKEY. 


\section{INTRODUCTION}

Chronic renal failure has recently become a common public health problem in the elderly population (1). Despite diagnostic and therapeutic advancements, the number of patients with endstage renal disease (ESRD) requiring renal replacement therapy is increasing every year. Therapeutic advancements and prolonged patient survival cause an increase in geriatric population in this patient group (2-3). Cardiovascular diseases are the most important cause of mortality and morbidity in patients with ESRD. The most common group of cardiovascular diseases observed in this patient group is atherosclerotic cardiovascular diseases (ASCVD) (4).

The epicardial adipose tissue is the adipose tissue found between the visceral pericardial layer of the heart and myocardium. The success of using the thickness of this tissue for determining cardiovascular risks has been demonstrated in many studies (5). Similarly, carotid intima-media thickness (CIMT) and femoral intima-media thickness (FIMT) are parameters that demonstrate the risk of subclinical atherosclerosis and successfully predict the risk of cardiovascular events in many studies (6-7).

Although the risk of ASCVD is increased in all patients with ESRD, it is not clear how this risk occurs in geriatric patients. Therefore, this study aims to evaluate the risks of subclinical atherosclerosis and cardiovascular events in geriatric patients with ESRD by evaluating epicardial fat thickness (EFT), CIMT and FIMT, which are considered to be the predictors of these risks.

\section{MATERIAL AND METHODS}

The study was designed as a cross-sectional study. The records of patients receiving chronic hemodialysis treatment at the outpatient clinic of hospital were examined. A total of 52 patients who started to receive chronic hemodialysis treatment after the age of 65 years and 51 healthy volunteers (control group) with similar demographic characteristics were included. The healthy participants had normal findings in treadmill test, myocardial perfusion scintigraphy, and coronary computed tomography (CT) that were conducted in the last one-year.

Exclusion criteria: Patients $<65$ years of age; those with known ASCVD (coronary artery disease, previous cerebrovascular event, or peripheral artery disease), patients who started to receive chronic hemodialysis treatment before the age of 65 years, hypertension, diabetes mellitus, known malignancy, chronic liver disease, connective tissue disease, hypothyroidism, hyperthyroidism, chronic steroid use, severe heart-valve disease or heart failure, history of heart surgery or smoking, familial hyperlipidemia, autoimmune disease, chronic renal failure and receiving peritoneal dialysis, and previous renal transplantation; or those with insufficient image quality or refusal to participate in the study were not included.

The participants in the patient group were diagnosed with chronic renal failure by a nephrologist according to the guidelines (8). Standard 2-dimensional (2D) echocardiography was performed by a cardiologist, and EFT was measured and recorded. CIMT and FIMT measurements were performed by radiologists. The selection of the patients and control group individuals was conducted by a nephrologist. Cardiologist and radiologist who measured EFT, CIMT and FIMT were blinded to the study.

Baseline demographic, anthropometric, and laboratory values of the participants in both groups were measured and recorded. EFT, CIMT and FIMT were recorded, and the differences between the two groups were evaluated for statistical significance.

Routine 2D echocardiography, conventional spectral Doppler ultrasonography, and epicardial fat thickness data

A Philips EPIQ 7 (Seattle, U.S.A) ultrasound system and Philips X5-1 (Seattle, U.S.A) probe (broadband transducer) were used for echocardiographic 
evaluations. The evaluations were performed using standard 2D and Doppler evaluations according to the recommendations of the American Society of Echocardiography and European Association of Cardiovascular Imaging. The ejection fraction (EF) was calculated using the modified Simpson method (9).

EFT is defined as the echo-free area between the free wall of the myocardium and the visceral layer of the pericardium. In this study, EFT was measured in the parasternal long-axis imaging at the end of the diastole and at three cardiac beats, perpendicular to the right ventricular free wall (Figure-1A) (10).

\section{Carotid and femoral ultrasonography}

A Siemens Acuson S1000 (Erlangen, Germany) ultrasound system and Siemens 9L4 (Erlangen, Germany) probe (broadband transducer) were used for ultrasonographic evaluations. CIMT was manually measured from the far walls of left and/or

Figure 1. Measurements of epicardial fat thickness on the free wall of the right ventricle from the parasternal long-axis views (A) and carotid (B) and femoral (C) intima-media thicknesses at the far wall of common carotid/femoral arteries.
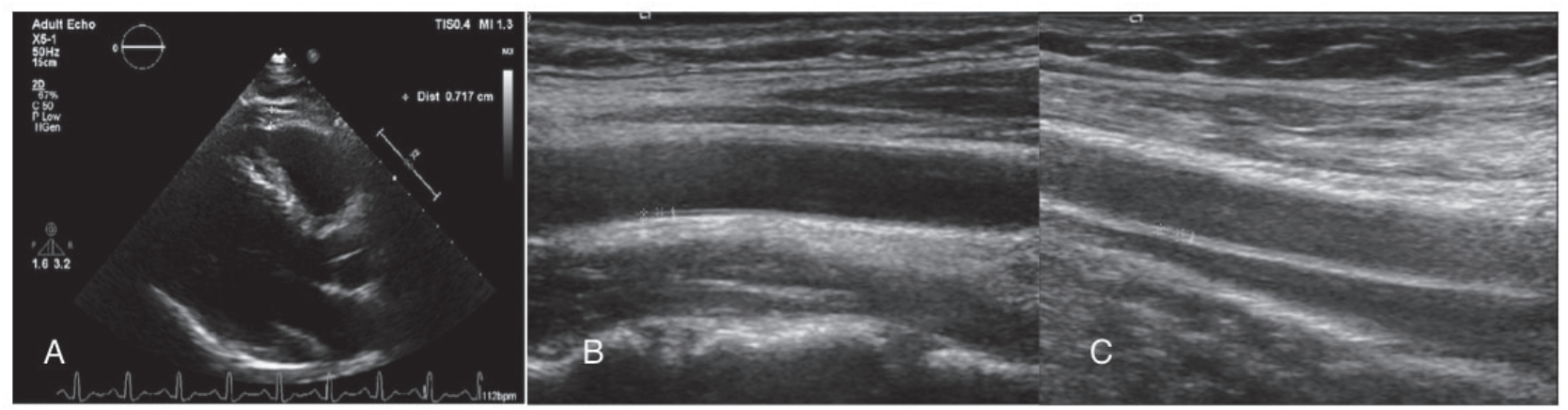

Figure 2. Comparison of carotid and femoral intima-media and epicardial fat thickness measurements between the groups.

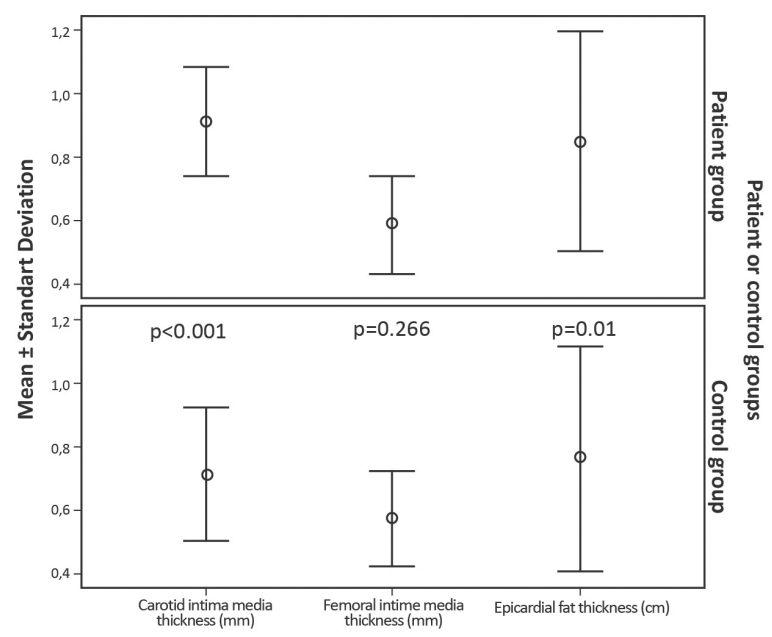

right common carotid arteries (opposite side of arteriyovenous dialysis fistula for patient group, both side for control group), at a region $10 \mathrm{~mm}$ proximal to the carotid bifurcation. Measurements were conducted on B-mode duplex ultrasound in a longitudinal plane. CIMT was measured on this image at three adjacent sites, $1 \mathrm{~mm}$ apart (Figure-1B). In addition, on B-mode duplex ultrasound, the mean FIMT at the far wall of the left or right common femoral arteries (opposite side of arteriyovenous dialysis fistula for patient group, both side for control group), was manually measured manually at three adjacent sites, $1 \mathrm{~mm}$ apart (Figure-1C).

The study was conducted in accordance with the criteria of the Helsinki Declaration, and approval was obtained from the local ethics committee (KA19/259). After providing detailed information about the study, written informed consent was obtained from all participants. 
Figure 3. Correlation analyses between carotid intima-media and epicardial fat thicknesses and the duration of dialysis.

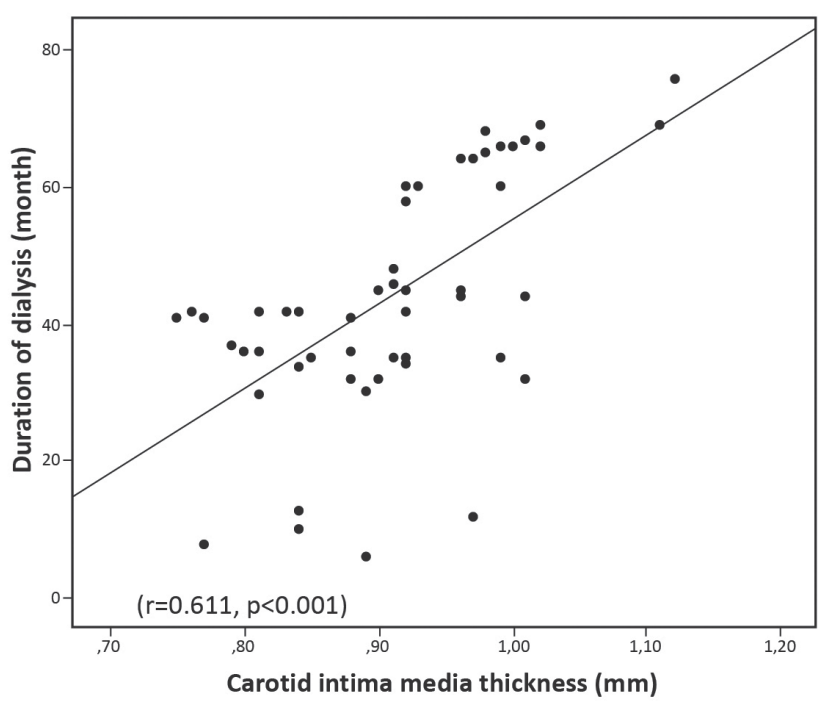

\section{Statistical analyses}

All parameters were evaluated using SPSS software (SPSS version 21.0; SPSS Inc., Chicago, IL, USA). Continuous variables were tested for normal distribution using Kolmogorov-Smirnov test. The results, including normally distributed variables, were expressed as mean \pm standard deviation; whereas on-normally distributed variables were expressed as medians and interquartile ranges. Categorical variables were shown as absolute values and percentages. Independent sample t-test was used to compare the two groups with respect to the variables with normal distribution and homogeneous group variance. The variables that showed a non-homogenous distribution were compared using the Mann-Whitney U-test. Categorical variables, such as clinical characteristics, of the groups were compared using the chi-square test. Spearman's or Pearson correlation tests were used to determine the degrees of association between continuous variables. Independent determinants of variables were determined using standard multiple linear regression analysis.

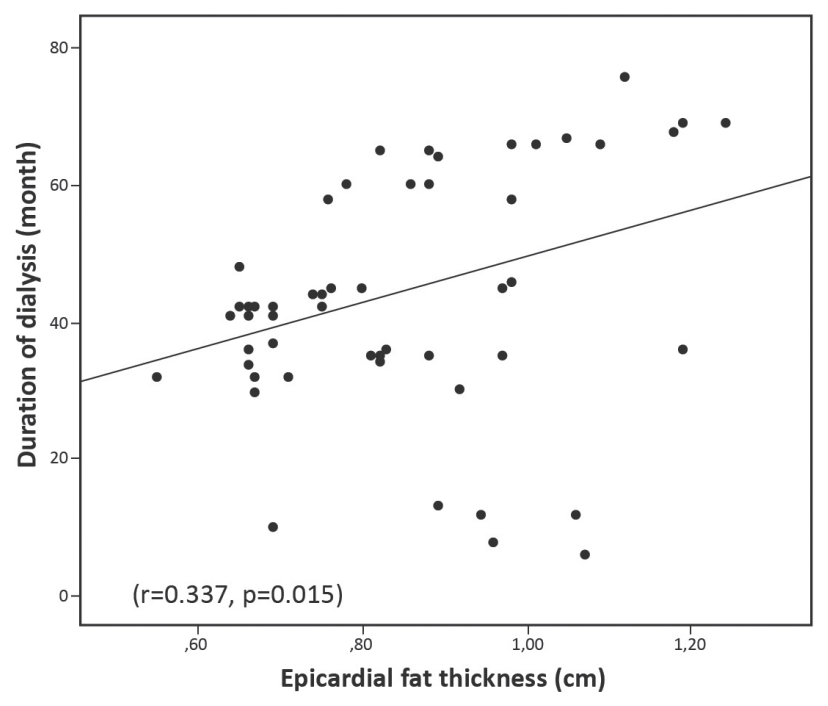

\section{RESULTS}

A total of 103 individuals, including 52 patients with ESRD (mean age: $73.92 \pm 5.63$ years) and 51 healthy volunteers (mean age: $74.49 \pm 4.63$ years), were included in this study. There was no statistically significant difference between patient and control groups in terms of baseline clinical characteristics $(p>0.05)$. The clinical characteristics of both groups are shown in Table I.

Both CIMT and EFT were significantly higher in patient group than in the control group $(0.91 \pm 0.08$ vs. $0.71 \pm 0.1 \mathrm{~mm}, p<0.001$ and $0.84 \pm 0.17$ vs. $0.75 \pm 0.17 \mathrm{~cm}, p=0.01$, respectively). However, there was no significant difference in FIMT between the two groups $(0.58 \pm 0.07$ vs. $0.56 \pm 0.97 \mathrm{~mm}, \mathrm{p}=266)$. Figure-2 shows the comparison of CIMT, FIMT and EFT measurements between the groups.

Table II shows the results of correlation analyses and linear regression analysis between age, body mass index, systolic blood pressure, diastolic blood pressure, fasting plasma glucose, EF, duration of dialysis, low-density lipoprotein (LDL), high-den- 
Table 1. Baseline clinical characteristics of the study population.

\begin{tabular}{|c|c|c|c|}
\hline & Patients $(n=52)$ & Control $(n=51)$ & $\mathbf{p}$ \\
\hline Age (years) & $73.92 \pm 5.63$ & $74.49 \pm 4.63$ & 0.579 \\
\hline Sex, female gender, n (\%) & $23(44.23)$ & $19(37.25)$ & 0.471 \\
\hline Body mass index $(\mathrm{kg} / \mathrm{m} 2)$ & $23.35 \pm 2.58$ & $23.49 \pm 2.28$ & 0.779 \\
\hline Hemoglobin (gr/dL) & $11.19 \pm 0.99$ & $11.47 \pm 0.87$ & 0.144 \\
\hline White blood cell (/mm3) & $7931 \pm 1131$ & $7841 \pm 1061$ & 0.678 \\
\hline Platelets $(100 / \mathrm{mm} 3)$ & $310(\mathrm{IQR}=53)$ & $315(I Q R=46)$ & 0.229 \\
\hline $\mathrm{Na}(\mathrm{mmol} / \mathrm{L})$ & $136.15 \pm 2.87$ & $136.24 \pm 2.77$ & 0.884 \\
\hline $\mathrm{K}(\mathrm{mmol} / \mathrm{L})$ & $3.94 \pm 0.34$ & $3.9 \pm 0.27$ & 0.553 \\
\hline EF (\%) & $55.33 \pm 2.94$ & $55.08 \pm 1.92$ & 0.613 \\
\hline $\mathrm{LDL}(\mathrm{mg} / \mathrm{dL})$ & $131.19 \pm 9.4$ & $131,65 \pm 10.17$ & 0.814 \\
\hline $\mathrm{HDL}(\mathrm{mg} / \mathrm{dL})$ & $42.23 \pm 3.64$ & $41.45 \pm 3.83$ & 0.292 \\
\hline Triglyceride (mg/dL) & $148(I \mathrm{QR}=60)$ & $141(\mathrm{IOR}=56)$ & 0.157 \\
\hline SBP $(\mathrm{mmHg})$ & $130.08 \pm 12.13$ & $127.25 \pm 11.71$ & 0.233 \\
\hline DBP $(\mathrm{mmHg})$ & $65 \pm 6.1$ & $62.84 \pm 7.76$ & 0.121 \\
\hline CRP (mg/L) & $2.76 \pm 1.18$ & $2.6 \pm 1.09$ & 0.474 \\
\hline Heart rate (beat/min) & $78.83 \pm 9.84$ & $75.55 \pm 10.26$ & 0.101 \\
\hline ALT (U/L) & $31.17 \pm 4.84$ & $30.69 \pm 4.87$ & 0.612 \\
\hline FPG (mg/dL) & $102.58 \pm 9.34$ & $101.92 \pm 9.67$ & 0.727 \\
\hline
\end{tabular}

ALT: Alanine aminotransferase, CRP: C-reactive protein, DBP: Diastolic blood pressure, EF: Ejection fraction, FPG: Fasting plasma glucose, HDL: High density lipoprotein, IQR: Interquartile range, K: Potassium, LDL: Light density lipoprotein, Na: Sodium, NA: Non available, SBP: Systolic blood pressure.

sity lipoprotein, triglyceride, hemoglobin, C-reactive protein, and CIMT and EFT measurements. Correlation analysis showed a statistically significant positive correlation between the duration of dialysis and CIMT and EFT, as shown in Figure-3 $(r=0.611, p<0.001$ and $r=0.337, p=0.015$, respectively). No significant correlation was observed between other variables. Moreover, in multiple linear regression analysis, there was a statistically significant relationship between the duration of dialysis and CIMT ( $\beta=0.657, p=0.001$ ). No significant relationship was found between other variables.

\section{DISCUSSION}

This is the first study that evaluates CIMT, FIMT and EFT in geriatric patients ESRD. Our study demonstrated that EFT and CIMT were significantly higher in the patient group than in the control group; however, there was no statistically significant difference between the two groups in terms of FIMT, suggesting indirect cardiovascular risks may be higher in geriatric patients with ESRD. However, the development of atherosclerosis in carotid arteries may occur earlier than the development of atherosclerosis in femoral arteries in this patient group. 
Table 2. Correlation and multiple linear regression analysis of epicardial fat and carotid intima media thicknesses and various clinical variables.

\begin{tabular}{|l|r|r|r|r|r|r|r|r|}
\hline & \multicolumn{4}{|c|}{ Correlation analysis } & \multicolumn{3}{c|}{ Multiple linear regression analysis } \\
\cline { 2 - 10 } & \multicolumn{2}{|c|}{ EFT } & \multicolumn{2}{|c|}{ CIMT } & \multicolumn{2}{|c|}{ EFT } & \multicolumn{2}{c|}{ CIMT } \\
\hline Variables & $\mathrm{r}$ & $\mathrm{p}$ & $\mathrm{r}$ & $\mathrm{p}$ & $\beta$ & $\mathrm{p}$ & $\beta$ & $\mathrm{p}$ \\
\hline Age & -0.148 & 0.296 & 0.103 & 0.469 & -0.038 & 0.784 & 0.027 & 0.851 \\
\hline BMI & 0.075 & 0.595 & -0.14 & 0.324 & 0.076 & 0.611 & -0.085 & 0.571 \\
\hline SBP & 0.215 & 0.126 & -0.087 & 0.538 & 0.124 & 0.557 & -0.113 & 0.59 \\
\hline DBP & 0.194 & 0.168 & -0.041 & 0.771 & 0.052 & 0.805 & -0.007 & 0.975 \\
\hline FPG & 0.116 & 0.413 & 0.241 & 0.085 & 0.08 & 0.589 & 0.264 & 0.075 \\
\hline EF & 0.139 & 0.325 & -0.142 & 0.315 & 0.019 & 0.911 & -0.016 & 0.926 \\
\hline DD & 0.337 & 0.015 & 0.611 & $<0.001$ & 0.32 & 0.072 & 0.657 & 0.001 \\
\hline LDL & 0.165 & 0.243 & -0.105 & 0.458 & 0.106 & 0.488 & -0.061 & 0.689 \\
\hline HDL & 0.257 & 0.066 & 0.23 & 0.101 & 0.097 & 0.643 & 0.175 & 0.409 \\
\hline TRG & 0.054 & 0.702 & 0.041 & 0.775 & 0.109 & 0.385 & 0.012 & 0.923 \\
\hline Hb & 0.094 & 0.507 & -0.07 & 0.624 & 0.094 & 0.507 & -0.493 & 0.624 \\
\hline CRP & 0.225 & 0.108 & -0.054 & 0.704 & 0.225 & 0.108 & -0.054 & 0.704 \\
\hline
\end{tabular}

BMI: Body mass index, CRP: C-Reactive protein, DBP: Diastolic blood pressure, DD: Duration of dialysis, EF: Ejection fraction, FPG: Fasting plasma glucose, Hb: Hemoglobin, HDL: High density lipoprotein, LDL: Light density lipoprotein, SBP: Systolic blood pressure, TRG: Triglyceride.

It has been known for many years that cardiovascular risk is increased in patients with chronic renal failure (11). In recent years, in addition to traditional risk factors, impaired renal function is considered to be a risk factor for coronary artery disease and cardiovascular event development (12). However, it is not clear why cardiovascular risks increase in patients with renal failure. Chronic inflammation, oxidative stress, and increased angiogenesis could be the reason for the increased risk of atherosclerosis in these patients (12). A study showed that proinflammatory cytokines are released more than epicardial fat tissue in patients with coronary artery disease (13). Many studies have demonstrated an increase in EFT in patients with ESRD (14-15). The cytokines released from the epicardial adipose tissue directly adjacent to the coronary arteries in the epicardial region may cause an increased risk of atherosclerosis and thus an increased risk of cardiovascular events. Oxidative stress is another mechanism responsible for increased risk other than infiltration. Previous studies have shown a positive correlation between EFT and serum oxidant content and a negative correlation with anti oxidant content (16-17).

Echocardiography, CT, and magnetic resonance imaging (MRI) are three different imaging methods that can be used to measure epicardial adipose tissue thickness. Each of the three imaging methods has its advantages and disadvantages. Among these methods, echocardiography is the most preferred method because it is economical and easily accessible and does not use radiation. The disadvantage, however, is that it is operator-dependent and allows only 2D evaluation. Moreover, it is impossible to obtain a healthy echocardiographic 
evaluation in obese people and individuals with poor image quality $(10,15,18)$. MRI is the gold standard method for the evaluation of epicardial fat. The advantages include the measurement of total adipose tissue, absence of radiation, and use of contrast media. The disadvantages are that it is expensive, not easily accessible, and cannot be used in patients with cardiac devices and implants. The advantages of CT, with high sensitivity and specificity in epicardial adipose tissue measurement, include allowing the measurement of total adipose tissue and a direct assessment of cardiovascular risk by calculating coronary calcium score. The main disadvantage is the use of radiation as well as nephrotoxic contrast agents in its application (18).

CIMT is another marker showing the risk of subclinical atherosclerosis. Its success in predicting cardiovascular events has been demonstrated by many studies (6). There are many mechanisms responsible for increasing the risk of atherosclerosis in ESRD, such as sharing the same risk factors, increased inflammation, renin-angiotensin and over-activation of the sympathetic system, and degradation of LDL oxidation (19).

Although MRI and CT can be used to evaluate carotid plaques and intima-media, ultrasonography is the most commonly used diagnostic method, which is considered to be a low-cost, noninvasive, and easily applicable imaging method. Carotid MRI, which has higher reproducibility than ultrasonography, has several disadvantages because it is expensive to conduct and not easily applicable. A good correlation has been shown between MRI and ultrasonography in the evaluation of wall area, thickness, and plaque index (20). CT is not a frequently preferred method for the evaluation of carotid plaque and intima media due to its in adequacy in evaluating dense calcified plaques and calcified plaques, low transfer of contrast media to lipid and fibrotic tissues, the presence of radiation, and use of contrast media. However, a study has shown that CIMT and carotid plaques can be evaluated using multi-detector row $\mathrm{CT}$ an- giography (21).

Different risk factors affecting the development of atherosclerosis may have different effects on different artery segments. For example, a study has shown that smoking and diabetes are more effective than other risk factors in the development of femoral atherosclerosis (22). In another study, hyperlipidemia and high blood pressure were found to be more associated with femoral atherosclerosis than with other risk factors (23). According to the results of our study, the development of femoral atherosclerosis in geriatric patients with ESRD may start later. Long-term prospective studies with more patients may be needed to evaluate this clearly.

\section{Study limitations}

This was a cross-sectional study, and therefore long-term results are currently unknown. Moreover, it was a single-centered study, with a relatively small number of patients; therefore, the study results should be confirmed with more patients and multi-centered studies. The epicardial adipose tissue was evaluated using 2D echocardiography; therefore, evaluation of total epicardial fat volume was not possible; however, studies have shown that $2 \mathrm{D}$ measurement in front of the right ventricle correlates with the total fat volume (10). In our study, although the operators performing ultrasonographic evaluations blindly evaluated the patient and control groups, the fact that these measurements are operator-dependent is another limitation of this study.

\section{CONCLUSION}

EFT and CIMT are significantly higher in geriatric patients with ESRD than in the control group. Therefore, cardiovascular risks may be higher in this patient group. However, no significant difference was observed between the two groups in terms of FIMT, suggesting that femoral atherosclerosis may develop later in patients with geriatric ESRD. 


\section{Conflict of Interest, Disclosure Statement}

The authors declare that they have no conflicts of interest. The authors have indicated they have no financial relationships relevant to this article to disclose

Ethical Approval: All procedures performed in this study involving human participants were in accordance with the ethical standards of the insti-

\section{REFERENCES}

1. Saran R, Robinson B, Abbott KC, et al. US Renal Data System 2018 Annual Data Report: Epidemiology of Kidney Disease in the United States. Am J Kidney Dis 2019;73(3S1):A7-A8. (PMID:30798791).

2. Murphy D, McCulloch CE, Lin F, et al. Centers for Disease Control and Prevention Chronic Kidney Disease Surveillance Team. Trends in Prevalence of Chronic Kidney Disease in the United States. Ann Intern Med 2016;165(7):473-81. (PMID:27479614).

3. Kesikburun B, Ekşioğlu E, Akdağ i, Çakıcı A. Determination of health-related quality of life in elderly hemodialysis patients. Turkish Journal of Geriatrics 2017;20(3):204-12.

4. Di Lullo L, House A, Gorini A, Santoboni A, Russo D, Ronco C. Chronic kidney disease and cardiovascular complications. Heart Fail Rev 2015;20(3):259-72. (PMID:25344016).

5. Ansaldo AM, Montecucco F, Sahebkar A, Dallegri F, Carbone F. Epicardial adipose tissue and cardiovascular diseases. Int J Cardiol 2019;278:25460. (PMID:30297191).

6. Carpenter M, Sinclair H, Kunadian V. Carotid Intima Media Thickness and Its Utility as a Predictor of Cardiovascular Disease: A Review of Evidence. Cardiol Rev 2016;24(2):70-5. (PMID:26825762).

7. Altin C, Sade LE, Gezmis E, Yilmaz M, Ozen N, Muderrisoglu $\mathrm{H}$. Assessment of epicardial adipose tissue and carotid/femoral intima media thickness in insulin resistance. J Cardiol 2017;69(6):843-50. (PMID:27613385).

8. Qaseem A, Hopkins RH Jr, Sweet DE, Starkey M, Shekelle P; Clinical Guidelines Committee of tutional and/or national research committee and with the 1964 Helsinki declaration and its later amendments or comparable ethical standards.

\section{Acknowledgments}

The authors would like to thank the cardiology and radiology fellows for their valuable contributions.

the American College of Physicians. Screening, Monitoring, and Treatment of Stage 1 to 3 Chronic Kidney Disease: A Clinical Practice Guideline From the American College of Physicians. Ann Intern Med 2013;159(12):835-47. (PMID:24145991).

9. Lang RM, Badano LP, Mor-Avi $V$, et al. Recommendations for cardiac chamber quantification by echocardiography in adults: an update from the American Society of Echocardiography and the European Association of Cardiovascular Imaging. J Am Soc Echocardiogr 2015;28(1):1-39. (PMID:25559473).

10. Sengul C, Ozveren O. Epicardial adipose tissue: a review of physiology, pathophysiology, and clinical applications. Anadolu Kardiyol Derg 2013;13(3):2615. (PMID:23395709).

11. Gansevoort RT, Correa-Rotter R, Hemmelgarn BR, et al. Chronic kidney disease and cardiovascular risk: epidemiology, mechanisms, and prevention. Lancet 2013;382(9889):339-52. (PMID:23727170).

12. Wang J, Wang F, Liu S, Zhou M, Zhang L, Zhao M. Reduced Kidney Function, Albuminuria, and Risks for All- cause and Cardiovascular Mortality in China: A Population-based Cohort Study BMC Nephrol 2017;18(1):188. (PMID:28592243).

13. Guzzardi MA, lozzo P. Fatty heart, cardiac damage, and inflammation. Rev Diabet Stud 2011;8(3):403-17. (PMID:22262077).

14. Aydın E, Altın C, Sakallıoğlu O, et al. Epicardial Adipose Tissue Thickness and Carotid Intima-Media Thickness in Hemodialysis Patients. Acta Cardiol Sin 2017;33(3):266-72. (PMID:28559657). 
15. Nakanishi K, Fukuda S, Tanaka A, et al. Epicardial Adipose Tissue Accumulation Is Associated With Renal Dysfunction and Coronary Plaque Morphology on Multidetector Computed Tomography. Circ J 2016;80(1):196-201. (PMID:26497330).

16. Abdallah E, El-Shishtawy S, Sherif N, Ali A, ElBendary $O$. Assessment of the relationship between serum paraoxonase activity and epicardial adipose tissue in hemodialysis patients. Int. Urol. Nephrol 2017;49(2):329-35. (PMID:27878751).

17. Macunluoglu B, Atakan A, Ari E, et al. Epicardial fat tissue thickness is correlated with diminished levels of co-enzyme Q10, a major antioxidant molecule among hemodialysis patients. Clin. Biochem 2014;47(1314):1231-4. (PMID:24882509).

18. Flüchter $S$, Haghi $D$, Dinter $D$, et al. Volumetric assessment of epicardial adipose tissue with cardiovascular magnetic resonance imaging. Obesity (Silver Spring) 2007;15(4):870-8. (PMID:17426322).

19. Palanca A, Castelblanco E, Betriu À, et al. Subclinical atherosclerosis burden predicts cardiovascular events in individuals with diabetes and chronic kidney disease. Cardiovasc Diabetol 2019;18(1):93. (PMID:31324183).
20. Zhang Y, Guallar E, Qiao Y, Wasserman BA. Is carotid intima-media thickness as predictive as other noninvasive techniques for the detection of coronary artery disease?.Arterioscler Thromb Vasc Biol 2014;34(7):1341-5. (PMID:24764454).

21. Saba L, Sanfilippo R, Montisci R, Mallarini G. Carotid artery wall thickness: comparison between sonography and multi-detector row CT angiography. Neuroradiology 2010;52(2):75-82. (PMID:19727693).

22. Paul TK, Chen W, Srinivasan SR, He J, Berenson GS. Contrast of the impact of multiple cardiovascular risk factors on the femoral and carotid intima-media thickness in asymptomatic young adults: the Bogalusa Heart Study. Atherosclerosis 2011;216(2):359-64. (PMID:21376319).

23. Kekalainen $P$, Sarlund $H$, Farin P, Kaukanen E, Yang $X$, Laakso M. Femoral atherosclerosis in middleaged subjects: association with cardiovascular risk factors and insulin resistance. Am J Epidemiol 1996;144(8):742-8. (PMID:8857823). 